\title{
EXISTENCE OF POSITIVE SOLUTIONS FOR A QUASILINEAR ELLIPTIC SYSTEM OF $p$-KIRCHHOFF TYPE
}

\section{QIN LI AND ZUODONG YANG}

Abstract. In this paper, we consider the existence of positive solutions to the following $p$ Kirchhoff-type system

$$
\begin{cases}-M\left(\int_{\Omega}|\nabla u|^{p} d x\right) \Delta_{p} u=g(x)|u|^{q-2} u+\left.\left.\frac{\alpha}{\alpha+\beta}|u|^{\alpha-2} u\right|^{\beta}\right|^{\beta}, & x \in \Omega, \\ -M\left(\int_{\Omega}|\nabla u|^{p} d x\right) \Delta_{p} v=h(x)|v|^{q-2} v+\frac{\beta}{\alpha+\beta}|u|^{\alpha}|v|^{\beta-2} v, & x \in \Omega, \\ u=v=0, & x \in \partial \Omega,\end{cases}
$$

where $\Omega$ is a bounded domain in $\mathbb{R}^{N}, M(s)=a+b s^{k}, \Delta_{p} u=\operatorname{div}\left(|\nabla u|^{p-2} \nabla u\right)$ is the $p$ Laplacian operator, $\alpha>1, \beta>1,1<p<q<\alpha+\beta<p^{*}=\frac{N p}{N-p}$.

Mathematics subject classification (2010): 35J65, 35J50.

Keywords and phrases: existence, quasilinear elliptic system, $p$-Kirchhoff type.

\section{REFERENCES}

[1] F. JÚlio, S.A. Correa, G.M. FigueIRedo, On an elliptic equation of p-Kirchhoff type via variational methods, Bull. Austral. Math. Soc, 74 (2006), 263-277.

[2] G. KIRChHOFF, Mechanik, Teubner, Leipzig, 1883.

[3] C.S. Chen, J.C. HUANG, L.H. LiU, Multiple solutions to the nonhomogeneous p-Kirchhoff elliptic equation with concave-convex nonlinearities, Applied Mathematics Letters, 26 (2013) 754-759.

[4] F. JÚlio, S.A. Correa, R.G. Nascimento, On a nonlocal elliptic system of p-Kirchhoff-type under Neumann boundary condition, Mathematical and Computer Modelling, 49 (2009), 598-604.

[5] G. Tarantello, On nonhomogeneous elliptic involving critical Sobolev exponent, Ann. Inst. H. Poincare Anal. Non Lineaire 9 (1992), 281-304.

[6] C.Y. CHEN, Y.C. KuO, T.F. WU, The Nehari manifold for a Kirchhoff type problem involving signchanging weight functions, J. Differential Equations, 250 (2011), 1876-1908.

[7] J.H. JIN, X. WU, Infinitely many radial solutions for Kirchhoff-type problems in $\mathbb{R}^{N}$, J. Math. Anal. Appl., 369 (2010), 564-574.

[8] D.M. CAO, H.S. ZHOU, Multiple positive solutions of nonhomogeneous semilinear elliptic equations in $\mathbb{R}^{N}$, Proc. Roy. Soc. Edinburgh Sect. A 126 (1996), 443-463.

[9] X.M. HE, W.M. Zou, Infinitely many positive solutions for Kirchhoff-type problems, Nonlinear Analysis, 70 (2009), 1407-1414.

[10] K. Perera, Z.T. Zhang, Nontrivial solutions of Kirchhoff-type problems via the Yang index, J. Differential Equations, 221 (2006), 246-255. 